\title{
Systematic review of interventions aimed at reducing hookah pipe use: Implications for practitioners and clinicians
}

\author{
Z Kader, ${ }^{1} \mathrm{MA}, \mathrm{CFS}$; N V Roman, ${ }^{1} \mathrm{PhD} ; \mathrm{R}$ Crutzen, ${ }^{2} \mathrm{PhD}$ \\ ${ }^{1}$ Child and Family Studies Unit, Faculty of Community and Health Sciences, University of the Western Cape, Cape Town, South Africa \\ ${ }^{2}$ Department of Health Promotion, Faculty of Health, Medicine and Life Sciences, Maastricht University/CAPHRI (Care and Public Health \\ Research Institute), The Netherlands
}

Corresponding author: Z Kader(zkader4@gmail.com)

\begin{abstract}
Background. Globally, tobacco ranks as one of the major risk factors for death, disease and disability. While strong measures have been implemented to reduce cigarette use, there are alternative ways to smoke tobacco, such as the hookah pipe. Hookah pipe use appears to pose a significant public health concern and has serious short- and long-term health consequences for users and those exposed to second-hand smoke. To date, few studies have reviewed hookah pipe interventions beyond the efficacy-based paradigm.

Objectives. To systematically review interventions aimed at reducing hookah pipe use through the RE-AIM framework (reach, efficacy, adoption, implementation and maintenance of results) in order to provide a practical means of evaluating interventions.

Methods. A systematic review spanning 12 databases identified studies aimed at reducing hookah pipe use. All methodological types of intervention studies that were peer reviewed and in the English language were considered for inclusion. The quality of each study was assessed. Ten studies were deemed eligible. For each study, data were extracted using the RE-AIM framework.

Results. All studies focused solely on the smoker, and their recruitment strategies were described. Eight studies reported meeting their objectives. Overall, the studies presented limited information regarding adoption success. The interventions were mainly supportive, educational or counselling sessions. Only five studies reported on the maintenance of results post intervention.

Conclusions. Interventions focusing on reducing hookah pipe use are limited. Counselling and educational support sessions seem to be the most feasible and potentially successful approaches for intervention.
\end{abstract}

S Afr Med J 2019;109(6):392-406 DOI:10.7196/SAMJ.2019.v109i6.13892

Tobacco is a global public health concern, ranking among the top three causes of death, disability and disease for most regions of the world, including Africa, the Middle East, Australia, southern Latin America, Eastern Europe and Asia. ${ }^{[1]}$ In South Africa (SA), one out of five people report using tobacco products. ${ }^{[2]}$ While strong measures have been implemented to reduce cigarette consumption, there are alternative ways of smoking tobacco; one of these is the hookah pipe. Unfortunately, this device does not minimise the burden but exacerbates it. SA studies have found that use of hookah pipes is highly prevalent among students ${ }^{[2]}$ and that it is initiated at a young age. ${ }^{[3]}$ There is a misconception that smoking tobacco through a hookah pipe reduces the potential harm from smoking, ${ }^{[4]}$ but in fact hookah pipe smokers may inhale an amount of smoke during one session that is equivalent to smoking 100 or more cigarettes. ${ }^{[5]}$ Recent research suggests that the hookah pipe is a gateway for use of other substances such as cannabis and alcohol. ${ }^{[6-9]}$

Moreover, smoking the hookah pipe can cause shortness of breath, nausea, vomiting, dizziness, fainting, headaches, coughing and loss of taste. ${ }^{[10,11]}$ Some of the longer-term effects include increased risk of periodontal disease, chronic obstructive pulmonary disease, lung cancer, nicotine dependence, oral cancer, low birthweight for babies of mothers who smoke hookah pipes during pregnancy, mouth ulcers and poor health-related quality of life. ${ }^{[12]}$ Some of these effects can strike after as little as one hookah smoking session. ${ }^{[5]}$ However, hookah smoking prevalence rates remain high. Hookah pipe use typically begins in adolescence, with $67 \%$ beginning to smoke in high school and only $26 \%$ beginning at university. ${ }^{[10]}$ The average age of onset of hookah pipe use is $\sim 17$ years, ${ }^{[8]}$ but children have been reported to have begun as early as age $10 \cdot{ }^{[3]}$ It is therefore important to include age groups in studies reviewing hookah pipe use to understand the extent of the problem.

In 2005, the World Health Organization released an advisory note about the growing concerns surrounding increased tobacco smoking using the hookah pipe ${ }^{[13]}$ More than a decade later, hookah pipe use is on the rise globally. There is a need for interventions specifically designed to prevent and control hookah pipe smoking, ${ }^{[14]}$ as proposed by several researchers focusing on tobacco research. ${ }^{[15-20]}$ However, in order to design an effective intervention, it is important to determine what interventions already exist. Drawing conclusions about their strengths and weaknesses will help researchers and practitioners improve existing approaches or develop new ones. While there are reviews on hookah pipe interventions, ${ }^{[21-24]}$ the present review extends the efficacy paradigm by extracting data using the RE-AIM framework, which assesses the reach, efficacy, adoption, implementation and maintenance of interventions, allowing researchers and practitioners to explore interventions at a deeper level than if their effectiveness alone was assessed. Current interventions on hookah pipe use the same methodology as reducing cigarette smoking and/or information sharing about health hazards. Research suggests that intervening in hookah pipe use may require alternative approaches, but efficacy studies alone may not help in developing these, as they mainly focus on the outcomes and not the properties of interventions.

\section{Objectives}

To systematically review interventions aimed at reducing hookah pipe use using the RE-AIM framework in order to put forward recommendations for clinicians and practitioners. 


\section{Methods}

The review was prepared according to PRISMA (Preferred Reporting Items for Systematic Reviews and Meta-analyses) standards. ${ }^{[25]} \mathrm{A}$ protocol was prepared in advance (it can be accessed at https://www. crd.york.ac.uk/prospero/display_record. php?RecordID=69514).

\section{Inclusion and exclusion criteria}

Studies were included based on the following criteria: (i) full-text studies in the English language; (ii) all types of intervention studies aimed at reducing hookah pipe use for any age group and in any setting; and (iii) singlegroup or multi-group trials of an intervention (or interventions) aimed at reducing hookah pipe use. Studies that were not in the English language and non-intervention studies (e.g. guidelines, protocols, discussion papers, reviews, editorials, legislation, identifying an intervention need, animal studies, and studies focusing solely on cigarettes and e-cigarettes and not on the hookah pipe) were excluded.

\section{Search strategy}

There was no specific search period, because the present study aimed to include as many hookah pipe intervention studies as possible. As the literature identifies age of onset as young as 10 years, preadolescents and adolescents were emphasised in the search terms. The following electronic databases were searched: Cinahl, Dentistry and Oral Sciences Source, GreenFILE, Health Source Consumer Edition, Health Source - Nursing/ Academic Edition, Medline, PsycARTICLES, SocINDEX, SPORTDiscus, Cochrane, Wiley and PubMed. Three sets of keywords relating to interventions and hookah pipe use were used, focusing on the following main keywords: (i) hookah pipe; (ii) interventions; and (iii) age group. Similar words were used within each set of keywords, for example: (i) shisha, narghile or waterpipe; (ii) strategies, treatment, therapy, best practice or programme; and (iii) preadolescents and adolescents. The same keyword variations were used for all 12 databases. In addition, the reference lists of the retrieved articles were manually searched for potentially eligible studies.

\section{Review procedure}

The review process consisted of three phases to identify appropriate studies to include in the present study. Phase 1 involved screening titles of the records, phase 2 involved screening of abstracts, and phase 3 involved reviewing full texts to ensure that they were eligible for the study. Lastly, reference lists of all eligible full texts were scrutinised for any more potential intervention studies that could be included. At each point, studies that did not meet the inclusion criteria were eliminated. Duplicates were manually sought and removed.

In total, 36344 titles were identified. After removal of duplicates $(n=143), 36201$ title records were screened, and the titles that did not meet the inclusion criteria were excluded. This screening process resulted in 31 abstracts being eligible for phase 2 (abstract review). The reference lists of these studies were reviewed and yielded 4 studies that were eligible for the present study. A total of 16 full-text articles were deemed eligible, but only 10 of these studies met the inclusion criteria. Six studies were excluded because 2 were interventions that focused solely on cigarette smoking, 1 was a study protocol, and 3 were not intervention studies. Fig. 1 shows the flow diagram of the review process described.

\section{Data extraction}

Data from the included studies were extracted and placed into a data extraction tool that was developed prior to the search and piloted. The following data were extracted: author, year, title, country and study design. This information provided a description of the studies. The data were then extracted according to the RE-AIM (reach, efficacy, adoption, implementation and maintenance of results) framework, as follows: reach (intended and reached target population); efficacy (effects of the intervention regarding hookah pipe use by determining the effect size using Cohen's $d$ or odds ratio); adoption (the extent to which target staff, venues or organisations adopted the intervention);

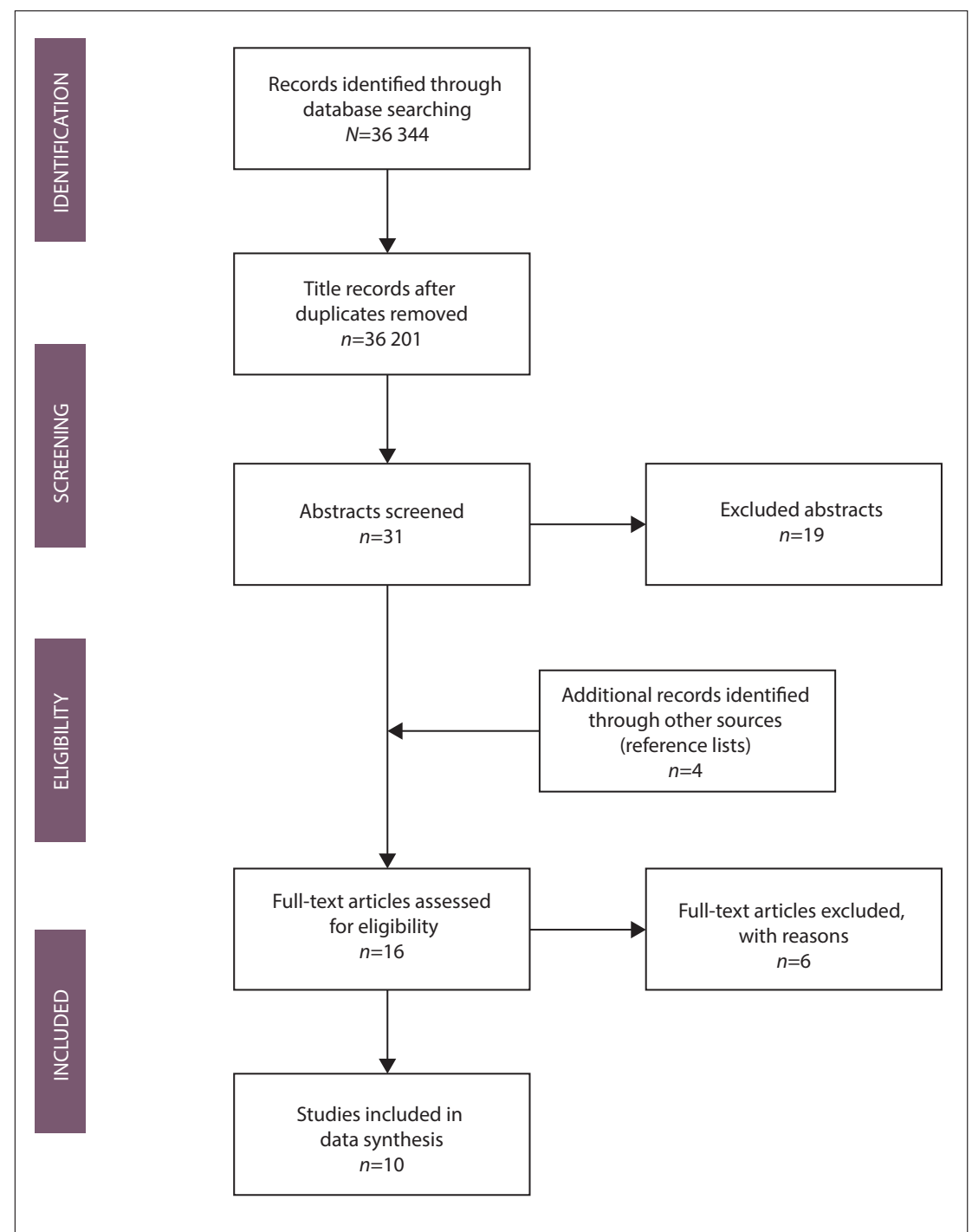

Fig. 1. PRISMA (Preferred Reporting Items for Systematic Reviews and Meta-analyses) flow diagram illustrating the review process (adapted from Moher et al. ${ }^{[25]}$ ). 
implementation (consistency and adaptation of implementing the intervention protocol in practice); and maintenance (intervention effects on individuals or settings over time, i.e. $>6$ months).

\section{Quality assessment}

All studies meeting the inclusion criteria underwent quality assessment using the adapted RE-AIM framework appraisal tool. ${ }^{[26]}$ The quality of the studies was rated using a percentage score on the five dimensions of the RE-AIM framework based on the content of the manuscripts. Each component was assessed and rated according to a three-grade scale: strong (67 - 100\%), moderate (34-66\%) and weak $(0-33 \%)$.

\section{Methodological quality of studies}

The methodological quality of the studies is described in Table 1. Eight studies reported moderately well in relation to their intervention to reduce hookah pipe use. Two studies had strong reporting in terms of the RE-AIM dimensions. The studies seemed to report extensively on efficacy but sparsely on maintenance of results. To obtain a global rating, the ratings were summed according to the guidelines of the quality assessment tool. ${ }^{[26]}$

\section{Data synthesis}

Narrative synthesis within the RE-AIM framework was used in this study. The quality of each study was based on the properties of each study according to the RE-AIM framework. The data were described on the basis of the reach, efficacy, adoption, implementation and maintenance aspects of the study.

\section{Results \\ Description of studies}

Sixty percent of the studies were from Asia, $30 \%$ from North America and $10 \%$ from Europe. There were no studies from Africa. The studies showed that the interventions were contextualised for these continents and settings. Of the 10 studies, 7 focused on the efficacy of the intervention, ${ }^{[27-33]} 2$ on the feasibility of the intervention, ${ }^{[34,35]}$ and 1 on describing the intervention. ${ }^{[36]}$ Fifty percent $(n=5)$ of the studies were of randomised controlled design, 30\% $(n=3)$ pre-test and post-test design, and $20 \%(n=2)$ quasi-experimental design. No interventions focused on preadolescents, but 2 studies included adolescents. ${ }^{[27,29]}$ The reasons for focusing on adolescents were that the period of adolescence has been identified as a higher risk factor for hookah pipe use than any other age group ${ }^{[2]}$ and that smoking often starts during adolescence. ${ }^{[27]}$ Some studies focused on adult smokers and included college or university students $\mathrm{s}^{[30,33,35]}$ and adults. ${ }^{[28,31,32,34,36]}$ Table 2 describes each intervention in terms of the RE-AIM framework.

\section{Reach}

All 10 interventions targeted the hookah pipe user. Participants were recruited through physician referrals, word of mouth, newspaper adverts, ${ }^{[34]}$ flyers, announcements, internet messages, social media, and entering college or university classes. ${ }^{[35]}$ Participants were also recruited door-to-door ${ }^{[36]}$ and via advertisements at the hospital where the study was conducted. ${ }^{[31]}$ Four studies made reference to their exclusion criteria, ${ }^{[29,31,32,34]}$ which were mainly severe medical conditions and inability to understand consent procedures. The inclusion criteria included age, and that participants needed to have attended high school, ${ }^{[27,29]}$ vocational school, ${ }^{[27]}$ college or university ${ }^{[30,33,35]}$ or the healthcare facility, ${ }^{[11,32]}$ be current smokers who smoked either one cigarette or hookah pipe per day ${ }^{[34]}$ or month ${ }^{[30]}$ be part of the US Air Force undergoing technical training, ${ }^{[28]}$ have access to the internet ${ }^{[35]}$ or have suspected pulmonary tuberculosis. ${ }^{[31]}$ The total enrolment of the 10 interventions was 13516 people.

When conducting intervention studies, participation or retention rates are usually a concern. Only 3 studies mentioned participation or retention rates, which were: (i) $99 \% ;{ }^{[36]}$ (ii) $82.2 \%$; ${ }^{[27]}$ and (iii) $37 \%$ of the participants completed all three in-person sessions, $40.7 \%$ completed all 5 telephonic sessions, and $26 \%$ completed all treatment sessions ( 3 in person and 5 telephonic sessions) in the intensive intervention arm; in the brief arm (i.e. less intensive intervention), $78.3 \%$ completed the single in-person session, $39.1 \%$ completed 3 telephonic sessions, and $34.8 \%$ completed all treatment sessions (1 in-person and 3 telephonic sessions). ${ }^{[34]}$ None of the studies discussed strategies for preventing loss to follow-up or encouraging retention of participants.

\section{Efficacy}

Two studies did not meet their objectives and stated that their intervention had little to no effect. ${ }^{[35,36]}$ However, 8 of the 10 studies $(80 \%)$ reported having met their objectives and displayed some degree of efficacy. ${ }^{[27-34]}$ Table 3 describes how effective the interventions were. Interventions were effective because they enhanced the social skills needed to avoid the use of drugs and improved the self-efficacy of the participants. The brief cessation treatment for hookah pipe smokers appeared feasible. Behaviour support interventions with or without bupropion achieved 6-month smoking abstinence among hookah pipe smokers. Modified perceptions of harm and addiction related to the hookah pipe and the intervention had a primary preventive effect on study participants. In one study, while the intervention helped prevent hookah pipe use, it was not effective in terms of convincing people who already smoked to quit. ${ }^{[27]}$ Furthermore, reports of decreased use and short- and long-term abstinence were not maintained. ${ }^{[33]}$

\section{Adoption}

Interventions were adopted in Iran, Syria, Pakistan, Israel, the USA, Egypt and Germany by physicians, paramedics, facilitators, research assistants, study staff and locals. Interventions were located online, ${ }^{[30]}$ at Berlin Lung Hospital ${ }^{[27]}$ and at colleges in the US Midwest that participants enrolled in the Quit and Win contest to quit smoking attended. ${ }^{[33]}$

For an intervention conducted in the community, requirements were a population between 10000 and 20000 persons; at least one primary, preparatory and secondary school; a public health clinic; a youth club and a mosque. ${ }^{[36]}$ Siddiqi et al..$^{[32]}$ needed to include a balance of urban and rural health centres, because the prevalence of cigarette smoking was higher in urban areas and hookah pipe use was more prevalent in rural areas. Prior to their intervention, Mohlman et $a l^{[36]}$ identified a need to engage with community leaders in the villages identified for intervention. Another intervention was developed in collaboration with tobacco experts, and tested over 4 months. ${ }^{[28]}$

\section{Implementation}

There were mixed findings in the reporting of implementation. Numbers of sessions ranged from 1 to 8 and duration of sessions from 10 minutes to 2 hours, while for the period of the implementation, interventions ranged from a 2-hour once-off session to a 12-month programme. The frequency of sessions was not described for any of the interventions. Most interventions focused on brief shortterm activity where participants entered voluntarily and provided 


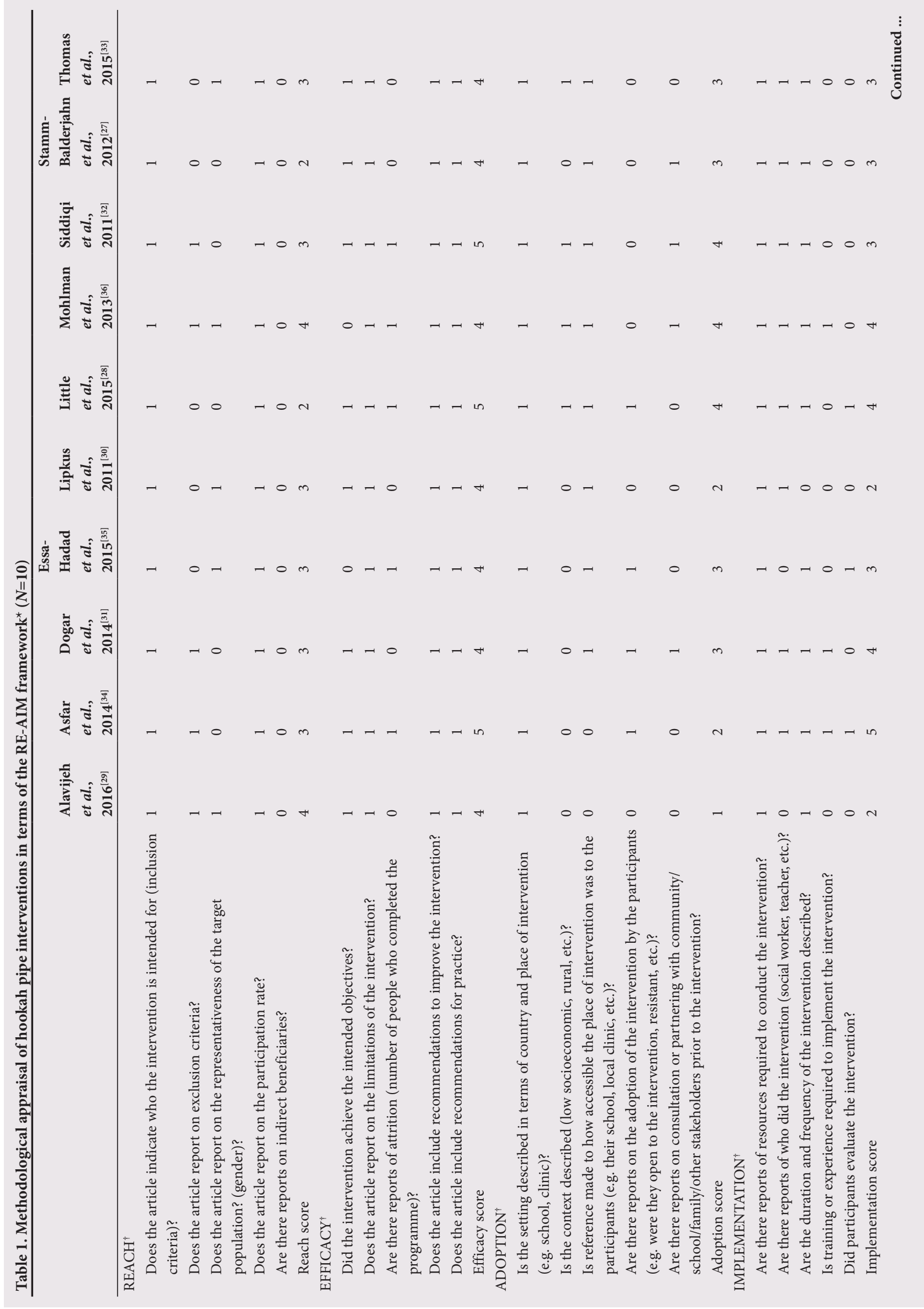




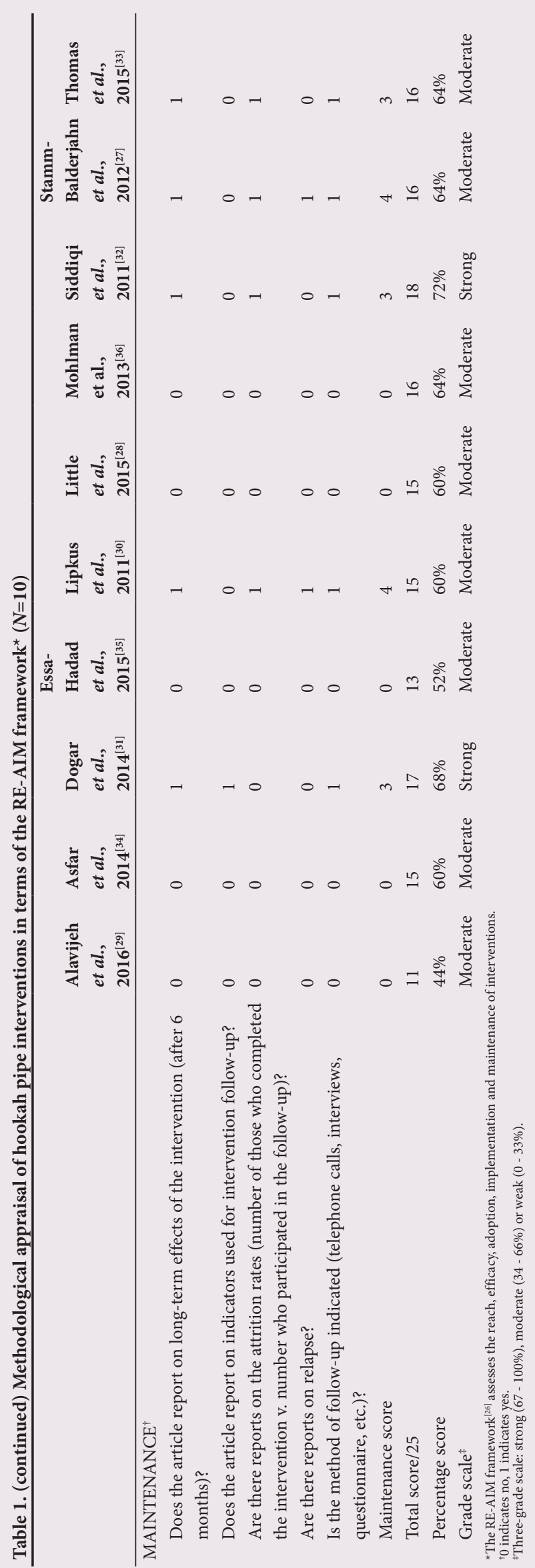

consent. Popular intervention sites included the local hospital ${ }^{[27]}$ and the communities where participants resided. ${ }^{[36]}$ The interventions were mainly supportive, educational or counselling, with or without medication. Sessions were conducted online, in person and telephonically. Interventions were implemented by physicians, nurses, auxiliary workers, study staff, paramedics or trained community members. Training ranged from 6 hours for physicians ${ }^{[34]}$ to a full day's training on intervention protocol and delivery tools for nurses and auxiliary nurses; ${ }^{[31]}$ in another study, locals were trained by the study staff so that they could implement and take responsibility for the intervention. ${ }^{[36]}$ In terms of resources and modalities, slide-show presentations, ${ }^{[27,29,30]}$ pamphlets, ${ }^{[29]}$ role-play, ${ }^{[29]} \mathrm{CDs},{ }^{[36]}$ booklets ${ }^{[36]}$ and self-help leaflets ${ }^{[32]}$ on smoking cessation were used.

Four studies incorporated an evaluation element to the intervention. ${ }^{[28,31,34,35]}$ Participants in one of these ${ }^{[34]}$ reported that the behaviour intervention was helpful because it encouraged physical activity, they received educational information, and they followed the rules of relapse prevention and received social support. Some participants reported that they preferred group counselling, while others preferred medication. ${ }^{[34]}$

\section{Maintenance}

Maintenance in the RE-AIM framework refers to whether outcomes were maintained at least 6 months post intervention. ${ }^{[19]}$ However, short-term intervention follow-up must also be considered, since this is also indicative of interventions reaching their goals. ${ }^{[37]}$ The period of follow-up as specified by 8 studies ranged from immediately post intervention to 6 months, but the latter was only the case in 5 studies. Three studies found that participants achieved prolonged abstinence at follow-up. ${ }^{[31,32,34]}$ At 1 month's follow-up in a further study, hookah pipe use decreased from $58.2 \%$ to $22.2 \%$ post intervention, and there was a slight increase in knowledge about the hookah pipe, but this increased knowledge was not found to be statistically significant. ${ }^{[35]}$ One study found little to no impact on the number of smokers and the amount of tobacco smoked among their participants. ${ }^{[36]}$ The studies made use of surveys, semi-structured interviews and analysis of urine cotinine to determine maintenance of results. Four studies reported attrition rates (percentage of participants at baseline who participated in follow-up), which ranged from $61 \%$ to $83 \% .{ }^{[32,34-36]}$ According to the findings presented, most of the interventions had a positive effect on participants. The studies did not report on the sustainability of the interventions. Two studies made reference to feasibility and found that the interventions were feasible. ${ }^{[34,35]}$

\section{Discussion and recommendations}

The objective of the present review was to assess all types of interventions aimed at reducing hookah pipe use in order to provide recommendations for practitioners and clinicians. This study provided an overview of what exists, what works and what can be built on to alleviate this public health concern. By reducing hookah pipe use, people can experience health, economic, environmental and family benefits because they will inhale fewer toxic substances; they can use their money for necessities or desires; there will be less environmental pollution; and families and peers will not be encouraged to smoke or be affected by passive smoke. ${ }^{[38,39]}$

When considering what exists, the interventions describe shortterm, prevention and early intervention support by means of supportive, educational or counselling sessions. All interventions targeted the smoker only, and family members or significant others were not invited to be part of the intervention. ${ }^{[27-34,36]}$ However, school-based prevention programmes and family-based intensive 


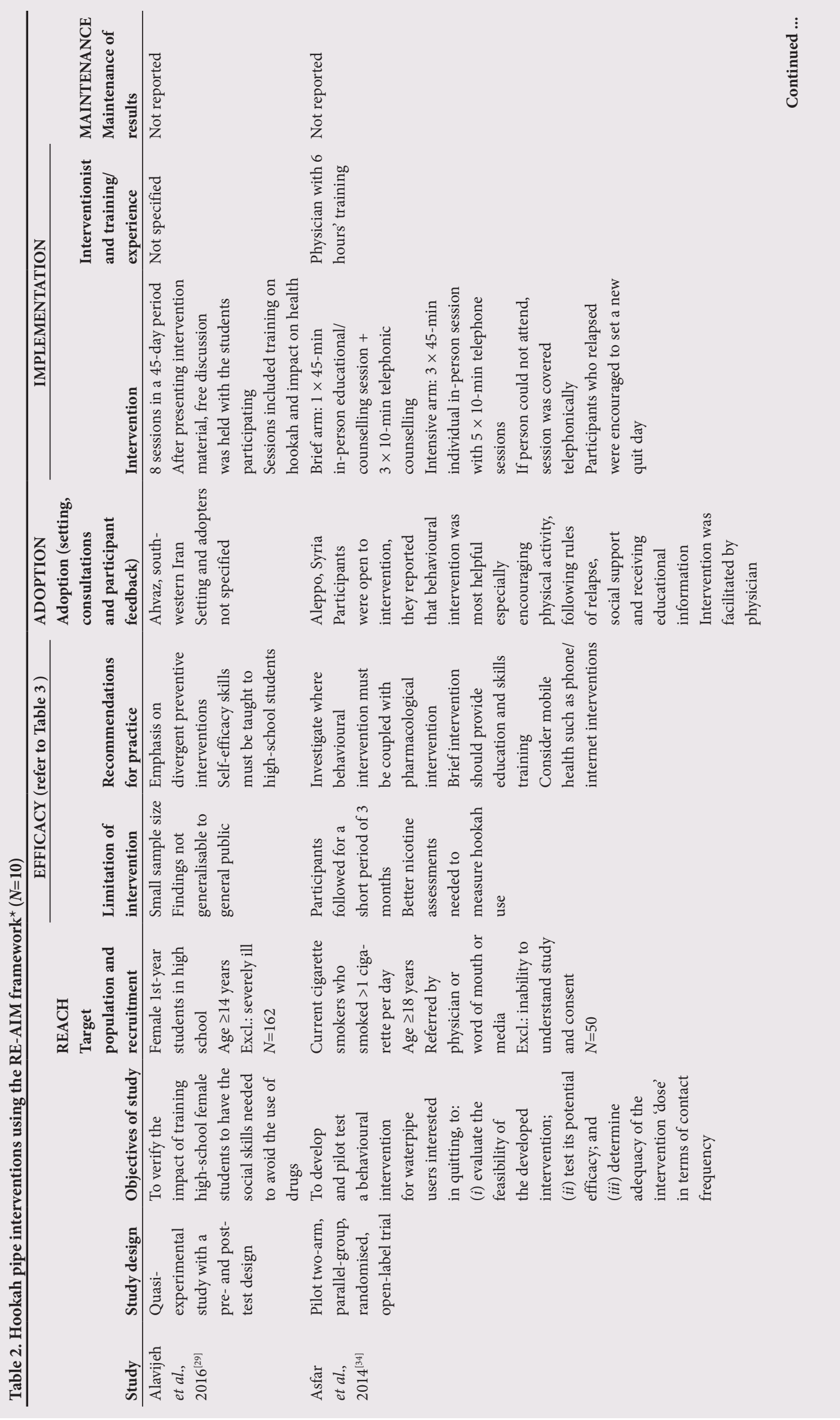




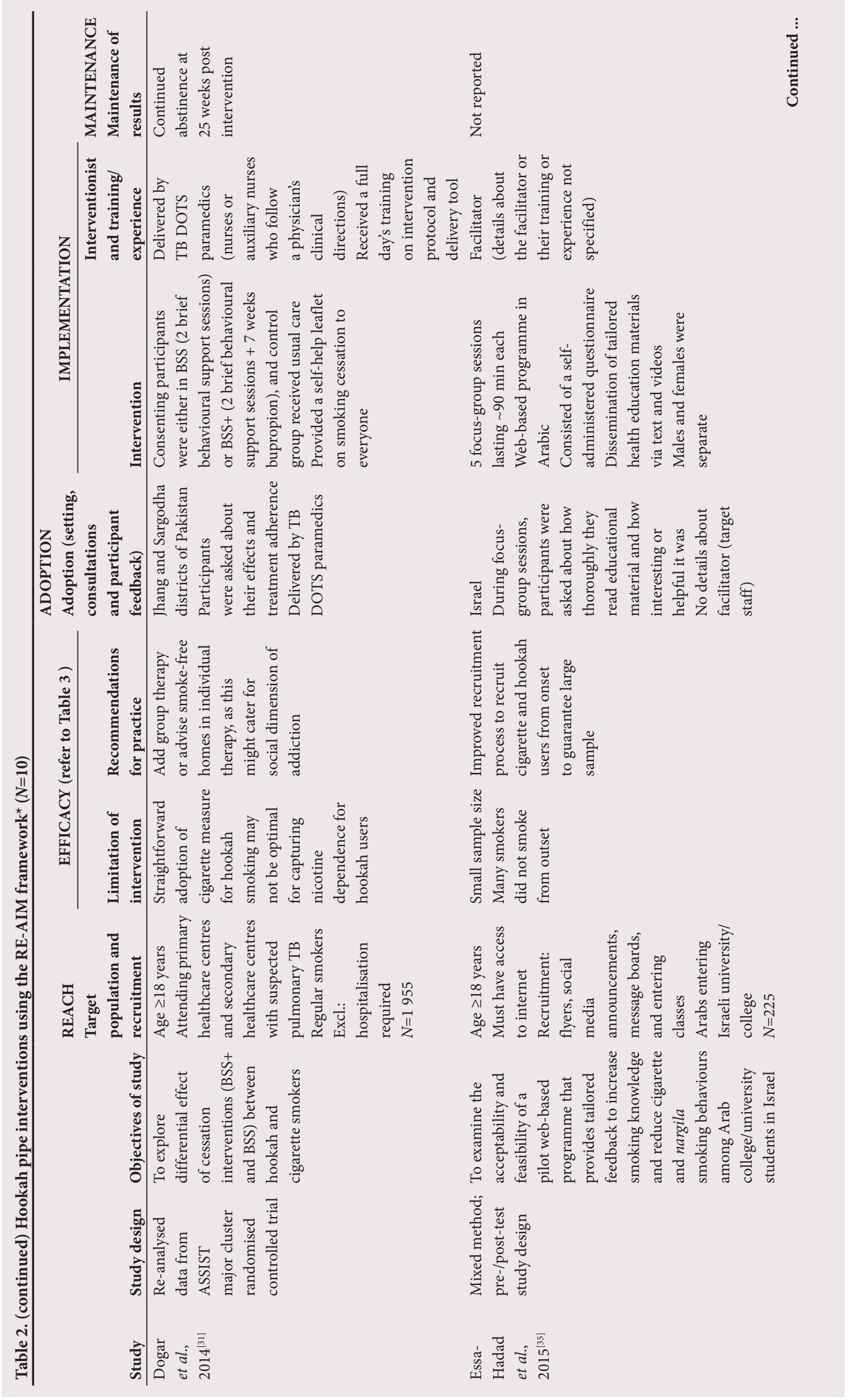




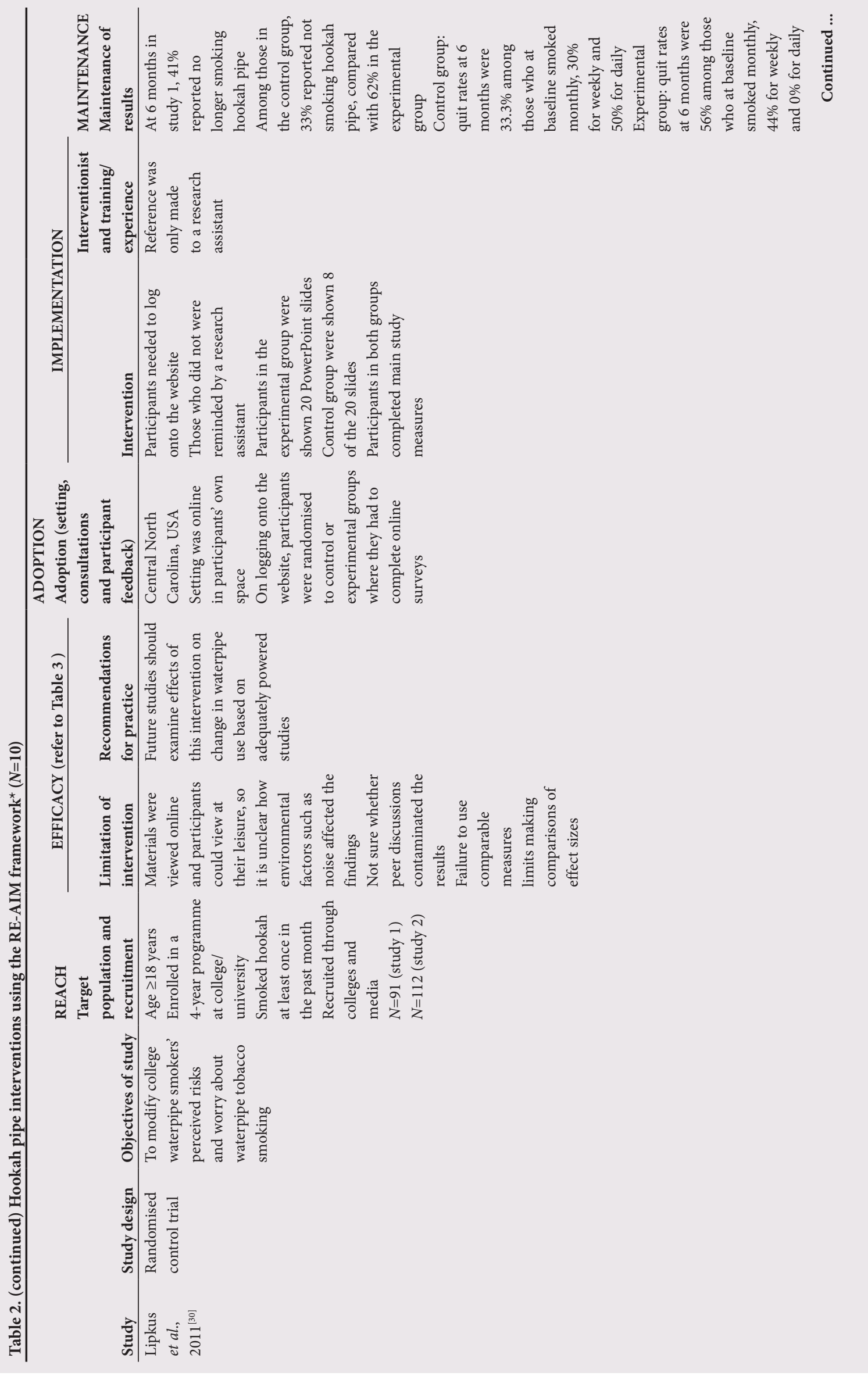




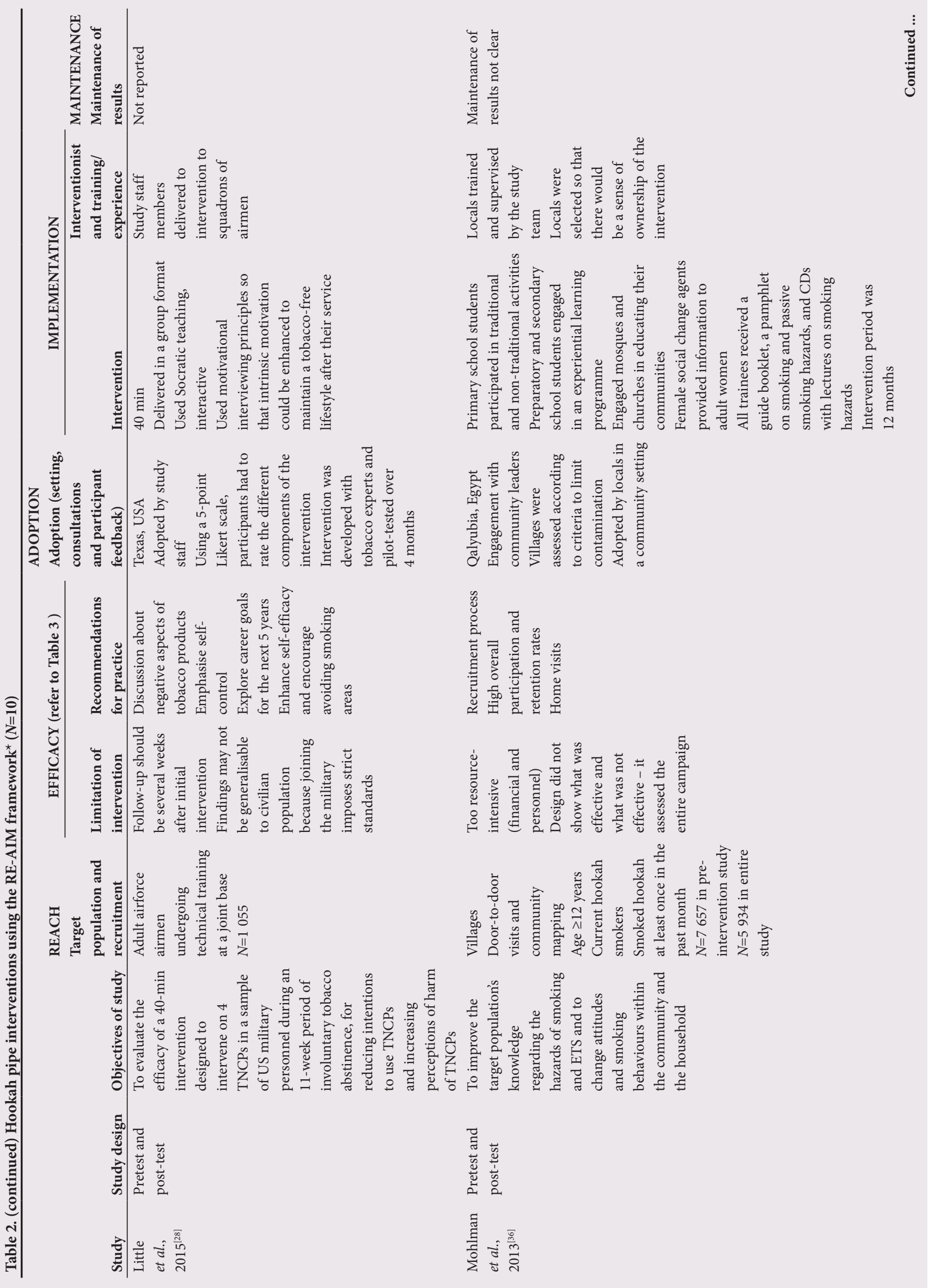




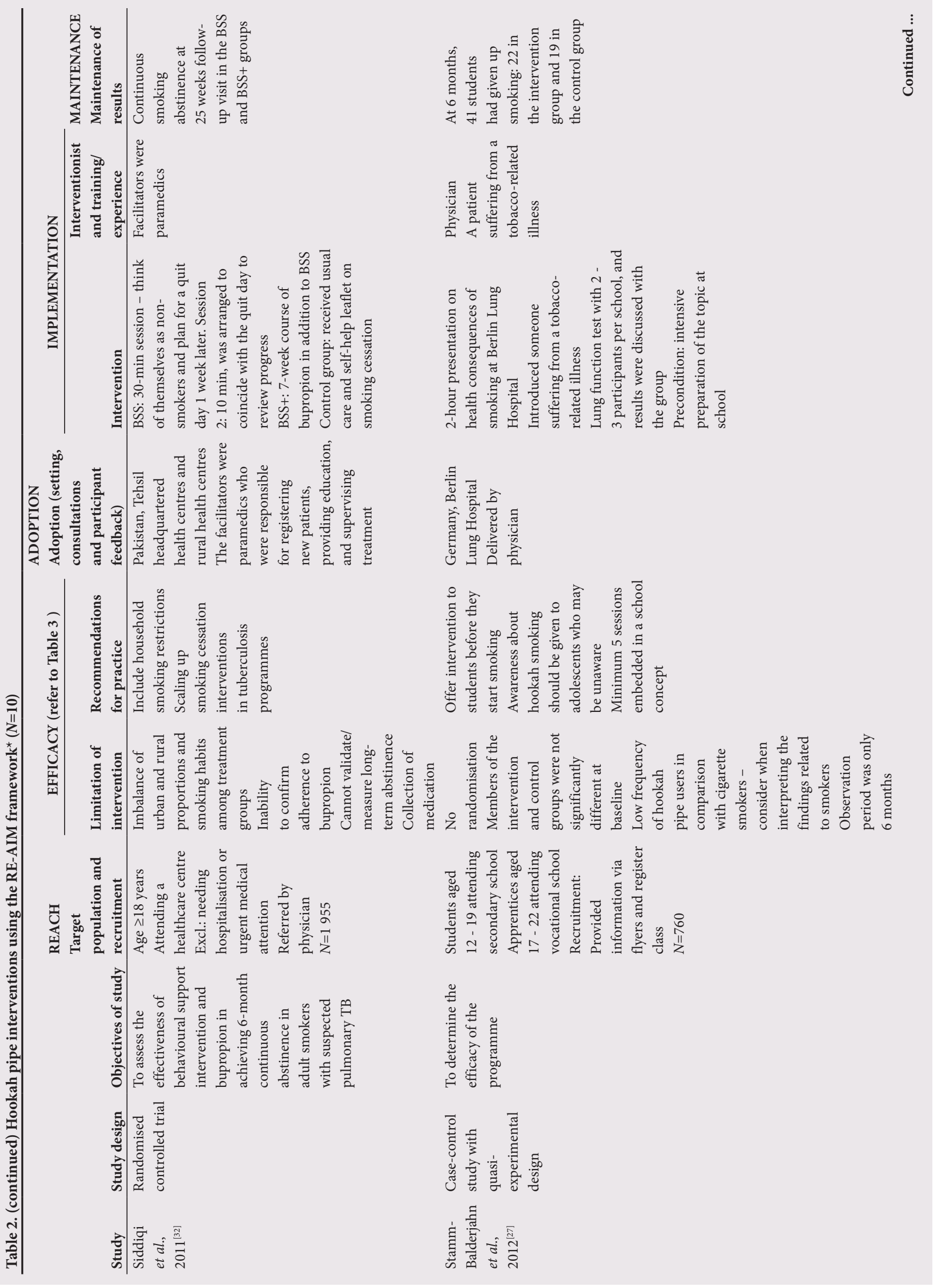




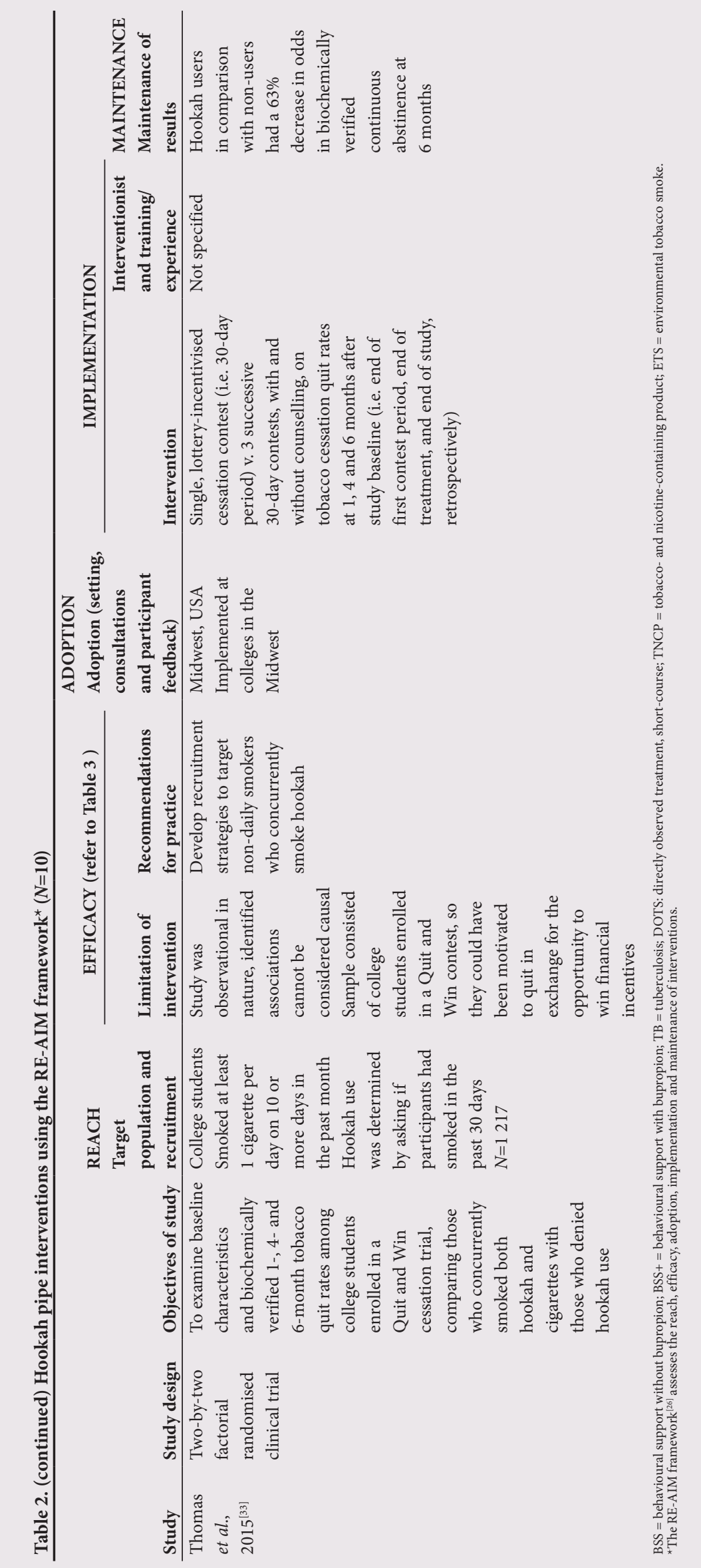

interventions focusing on family functioning have also shown promise. ${ }^{[27,29,40]}$ None of the 10 intervention studies included in this review focused on a family model, and this could potentially be a gap. Most of the interventions either aimed to change perceptions and behaviour or used the same interventions that are used for cigarette smoking abstinence, or ones similar to these. This approach is advised against, as hookah pipe smoking has its own characteristics and unique features that make it appealing. ${ }^{[13]}$ It is recommended that interventions should target these features, such as the attractive aroma, the taste, the pleasant bubbling sound, the social atmosphere, and the bonding and sharing over a hookah pipe. ${ }^{[13]}$ Moreover, hookah pipe users believe that smoking is relatively harmless compared with use of other substances, so awareness campaigns that clearly emphasise how hookah smoking affects health and wellbeing, including that of the wider community, are needed. ${ }^{[13]}$

When considering what works, none of the studies reported that recruiting participants was a challenge, which implies that the recruiting strategies described are likely to yield positive results if used in future interventions. Interventions were primarily concerned with improving health by encouraging participants to abstain. However, hookah pipe use also affects the economy and the environment, and researchers and practitioners should take this into account when planning interventions. The studies reported that the intervention settings were familiar locations such as the home or a local clinic, church or school. Use of these settings made the interventions accessible to participants, which is important, especially in low socioeconomic contexts where people may not have the resources to travel far for an intervention. This factor could explain why retention rates were relatively high. Community members should be involved in the design of the intervention, to generate community support, commitment and interest. ${ }^{[36,41]}$ It is also important for them to provide input on details such as the venue. A clinic, for example, may be easily accessible, but people could feel stigmatised attending meetings there. ${ }^{[41]}$ Involving the community where the intervention will occur is especially important when trying to change a specific behaviour that ultimately affects the health of the public, and will also encourage communities to adopt the programme and make it more sustainable. The interventions described were generally short term and shown to be effective during the intervention, implying that the resources and modalities employed worked. However, there is little evidence to indicate whether the effects would last over time or if the interventions were only effective for their duration. This 


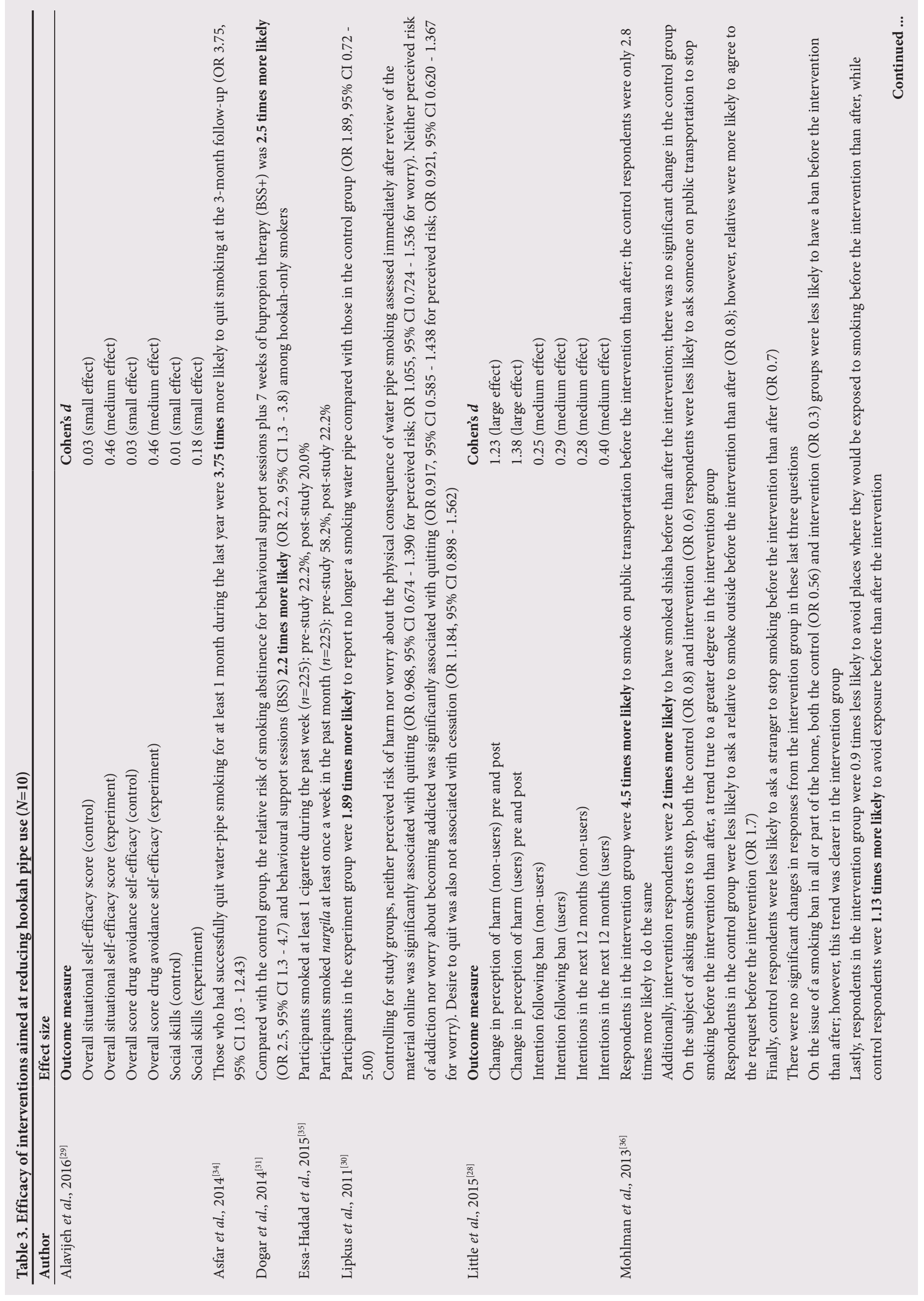




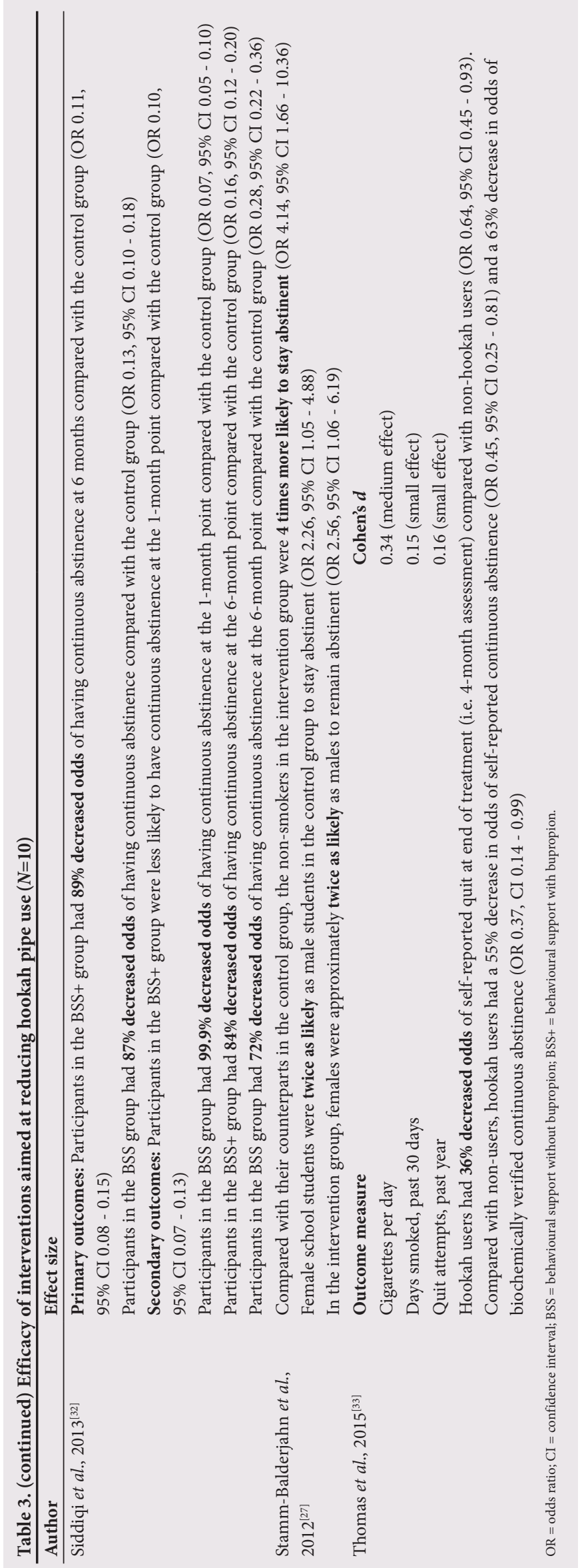

issue is evidenced by studies that found decreased odds of staying abstinent at 4 months and 6 months post intervention. ${ }^{[32,33]}$ It appears that brief interventions work, but failure to follow up is not conducive to maintaining the results. Cessation interventions are feasible and effective, but continuation beyond the implementation phase is unfortunately rare. ${ }^{[42]}$ Lack of follow-up or continuation of care can be attributed to organisational factors, funding and demand for service, hospital or school culture and community responsibility, but measures need to be put in place to enable follow-up, or the likelihood of results being sustained is slim.

While all 10 interventions showed promise, an opportunity exists to build on existing interventions. The present study has shown that no interventions to reduce hookah pipe use in Africa have been reported or evaluated, and we therefore present the following recommendations for clinicians and practitioners operating in resource-constrained settings, such as those typical of Africa. However, these suggestions are not limited to resource-constrained settings, and they may be applied in other contexts if it is feasible to do so.

- As information and interventions related to hookah pipe use and treatment are still in their early stages, it is advisable that clinicians and practitioners should attend training and familiarise themselves with hookah pipe research. This will give them a clear understanding of how hookah pipe use differs from that of cigarettes and other tobacco products, so that they can advise and intervene appropriately.

- Governments also have an important role to play in the reduction of hookah pipe use, and awareness and information sessions should therefore be lobbied with government departments so that they can be prioritised in budgets in order to provide the service at schools, workplaces, clinics and community centres.

- Banning hookah pipes in public spaces and in the company of children is important to protect users: (i) from peers who may negatively influence them to add substances or use other substances concurrently; (ii) from being labelled; and (iii) from being caught by law officials, community leaders or parents, which may lead to dire consequences such as punishment, embarrassment or harassment. Banning smoking in public places is crucial for nonusers too, as they may experience health problems from secondhand smoke and may be influenced by, exposed to or coerced into hookah pipe smoking.

- There should be stricter regulations and adherence to policies on the purchase of hookah pipes, tobacco and coals, as well as on advertising and display. In SA, the Tobacco Control Bill of 2018 $8^{[43]}$ identifies the hookah pipe as a tobacco product. The Bill states that retailers may not display the tobacco product at the place of business but may make the product available to consumers over the age of 18 years. Furthermore, the Minister must prescribe standardised packaging and labelling of tobacco products in terms of colour, texture, size, manufacturers' details, tax stamps and health warnings. The Bill further stipulates that no person shall advertise or promote or cause any other person to advertise or promote tobacco products.

- As people view hookah pipe use as relatively harmless, awareness campaigns should occur in various settings such as schools and clinics and different communities. A roadshow involving hookah pipe users from different ages and backgrounds may be a possibility. A variety of actors would be needed, so that the show can appeal to a wide range of audiences. For example, if the road show is aimed at adolescents, there should be adolescent actors so that it is seen as peer-based and relevant. At this point, recruitment could occur by means of methods described in this review. Intervention should follow soon after. 
- Many people do not have the time or resources to stay out of school or work for long periods of time, so mutually convenient times and locations must be arranged.

- Emphasis should be placed on assertion training and skills training to decline hookah pipe use, and being comfortable with the consequences of saying 'No'.

- It has been noted that counselling and educational support sessions are the most feasible, so mental health practitioners should be involved to provide counselling if hookah pipe use is related to coping with stress, trauma or challenges within the family.

- Doctors and nurses play an instrumental role in teaching about both the short- and long-term health effects, which must be described in a way that is relevant to the subjects' interests and age groups and that makes the information understandable and relatable. For example, telling adolescents that they may get cancer when they are older may not be as effective as informing them how hookah smoking affects their ability to play football (if that is their interest). Similarly, telling a pregnant mother how hookah smoking may affect her unborn child would be more meaningful than informing her about its negative effects on the environment.

- As hookah pipe use is a social phenomenon, a social element needs to be included when planning the intervention. Involving family members should be encouraged. Reasons why people use the hookah pipe should be explored; this may be done in individual or group counselling sessions. Once this understanding has been reached, it will be easier to educate, intervene or refer appropriately.

- It is very important that interventions be adapted to local culture, language and settings. Practitioners and clinicians must be cognisant about potential challenges (such as poor turnout, resistant beneficiaries, etc.) that may arise when attempting to intervene, and a plan to mitigate these challenges should be in place prior to the intervention.

- Most importantly, effective monitoring and evaluation strategies must be applied to measure progress over time to ensure the maintenance of results.

\section{Proposals for research}

It is necessary for interventions to be studied in terms of their impact over time, including health, social, economic and environmental effects. It would also be interesting to compare interventions and attitudes to hookah pipe use in low socioeconomic communities, middle-income communities and high socioeconomic communities to determine how needs vary according to context. Comparisons can be made of interventions aimed at reducing cigarette smoking $v$. hookah smoking, to establish whether the same approach works for both or a different approach is needed for each practice. There is a clear need for rigorously designed interventions focusing on reducing hookah pipe use to be published and disseminated. Studies should focus on why people enjoy smoking the hookah pipe and what needs it satisfies.

\section{Study limitations}

Although the current review utilised a broad search category and 12 databases, only trials published in journals within the included databases were located, thereby yielding only 10 appropriate studies. Owing to the heterogeneity of the identified studies, it was challenging to compare them in terms of strengths and weaknesses for the RE-AIM dimensions. Not all studies provided sufficient information about the intervention, its effectiveness and its impact, making it difficult to discuss the sustainability of the interventions. Some studies included cigarette smoking and hookah smoking, so it was not possible to make conclusions on the hookah interventions specifically. Moreover, while the RE-AIM framework is a model that can be used to assess properties of various interventions and their effectiveness, several other models exist and could have provided different insights - for example, cost implications, scalability, feasibility and replicability of the interventions.

\section{Conclusions}

The present systematic review indicates that there are limited quality interventions globally that focus on reducing hookah pipe use, and none in Africa. However, by drawing from strengths and weaknesses of existing interventions and incorporating the recommendations for future ones, development of a means to reduce hookah pipe use may be on the horizon. Counselling and educational support sessions seem to be the most feasible and potentially successful approaches, but more work is necessary. The time for action is now, and all practitioners and clinicians need to play a role in intervening in this major public health concern that is spreading over not only our country and continent but also the globe. We consider that this review is a good starting point that can contribute to the design of and decision-making regarding effective public health interventions to reduce hookah pipe use.

Declaration. This article was part of ZK's $\mathrm{PhD}$ project on designing an intervention to reduce hookah pipe use by adolescents, for which publication was a requirement.

\section{Acknowledgements. None.}

Author contributions. ZK was responsible for the conception, design, implementation, data analysis and drafting the article. NVR and RC were responsible for review and revision of the article.

Funding. This work was supported by the Health and Welfare Sector Education and Training Authority Sciences (grant no. 17/PGRB/L000335) and the National Institute for the Humanities and Social Sciences (grant no. SDS17/1878).

Conflicts of interest. None.

1. Lim SS, Vos T, Flaxman AD, et al. A comparative risk assessment of burden of disease and injury attributable to 67 risk factors and risk factor clusters in 21 regions, 1990 - 2010: A systematic analysis for the Global Burden of Disease Study 2010. Lancet 2012;380(9859):2224-2260. https://doi.org/10.1016/ S0140-6736(12)61766-8

2. Ware LJ, Charlton K, Kruger R, et al. Assessing tobacco use in an African population: Serum and urine cotinine cut-offs from South Africa. Drug Alcohol Depend 2018;195:82-89. https://doi.org/10.1016/j. drugalcdep.2018.11.022

3. Combrink A, Irwin N, Laudin G, Naidoo K, Plagerson S, Mathee A. High prevalence of hookah smoking among secondary school students in a disadvantaged community in Johannesburg. S Afr Med J 2010;100(5):297-299. https://doi.org/10.7196/SAMJ.3965

4. Ray CS. The hookah - the Indian waterpipe. Curr Sci India 2009;96(10):1319-1323. https://www.jstor. 4. Ray CS. The hookah - the Indian waterpipe. Curr Sci India 2009;96(10):1319.
org/stable/24105369? seq=1\#page_scan_tab_contents (accessed 29 April 2019).

5. Folan P, Fardellone C, Abamova R; Tobacco Control Committee of the American Thoracic Society. 5. Folan P, Fardellone C, Abamova R; Tobacco Control Committee of the American Thoracic Society.
Hookah pipes. Am J Respir Crit Care Med 2018;198:P17-P18. https://wwwatsjournals.org/doi/ pdf/10.1164/rccm.1989P17 (accessed 29 April 2019).

6. Kandel D, Kandel E. The gateway hypothesis of substance abuse: Developmental, biological and societal perspectives. Acta Paediatr 2015;104(2):130-137. https://doi.org/10.1111/apa.12851

7. Jacobs L, Roman NV, Schenk C. Gender performativity and addiction within the family: Focus on the hookah. Gender Behav 2015;13(2):6753-6759. https://journals.co.za/content/genbeh/13/2/EJC182548 (accessed 29 April 2019)

8. Roman NV, Schenck C, Jacobs L, September SJ. Hookah use: Could families be a risk factor for future addiction? J Child Adoles Subst 2017;26(1):11-17. https://doi.org/10.1080/1067828x.2016.1175985

9. Fielder RL, Carey KB, Carey MP. Hookah, cigarette, and marijuana use: A prospective study of smoking behaviors among first-year college women. Addict Behav 2013;38(11):2729-2735. https://doi. org/10.1016/j.addbeh.2013.07.006

10. Van der Merwe N, Banoobhai T, GqwetaA, et al. Hookah pipe smoking among health sciences students. S Afr Med J 2013;103(11):847-849. https://doi.org/10.7196/SAMJ.7448
S

11. Smith-Simone S, Maziak W, Ward KD, Eissenberg T. Waterpipe tobacco smoking: Knowledge, . Smith-Simone S, Maziak W, Ward KD, Eissenberg T. Waterpipe tobacco smoking: Knowledge,
attitudes, beliefs, and behavior in two US samples. Nicotine Tob Res 2008;10(2):393-398. https://doi. org/10.1080/14622200701825023

12. World Health Organization Study Group on Tobacco Product Regulation. Advisory Note: Water Pipe Tobacco Smoking: Health Effects, Research Needs and Recommended Actions for Regulators 2015. 2nd ed. Geneva: WHO, 2015. http://apps.who.int/iris/bitstream/handle/10665/161991/9789241508469. eng.pdf;jsessionid=F98AC171773747B05E98A9BA5C1346F6? sequence=1 (accessed 8 January 2019).

13. World Health Organization Study Group on Tobacco Product Regulation. Advisory Note: Water Pipe Tobacco Smoking: Health Effects, Research Needs and Recommended Actions for Regulators. Geneva: WHO, 2005. http://apps.who.int/iris/bitstream/handle/10665/161991/9789241508469_eng.pdf;jsessioni

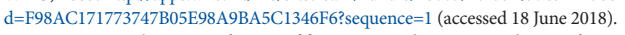

14. Lopez AA, Eissenberg T, Jaafar M, Afifi R. Now is the time to advocate for interventions designed specifically to prevent and control waterpipe tobacco smoking. Addict Behav 2017;66:41-47. https://doi. org/10.1016/j.addbeh.2016.11.008 
15. Maziak W, Eissenberg T, Klesges RC, Keil U, Ward KD. Adapting smoking cessation interventions for developing countries: A model for the Middle East. Int J Tuberc Lung Dis 2004;8(4):403-413.

16. Giuliani KK, Mire OA, Jama S, et al. Tobacco use and cessation among Somalis in Minnesota. Am J Prev Med 2008;35(6):457-462. https://doi.org/10.1016/j.amepre.2008.09.006
M.

17. Toghianifar N, Sarrafzadegan N, Roohafza H, Sadeghi M, Eshrati B, Sadri G. Smoking cessation support Toghianifar N, Sarrafzadegan N, Roohafza H, Sadeghi M, Eshrati B, Sadri G. Smoking
in Iran: Availability, sources and predictors. Indian J Med Res 2011;133(6):627-632.

18. Shihadeh A. Investigation of mainstream smoke aerosol of the argileh water pipe. Food Chem Toxicol 2003;41(1):143-152. https://doi.org/10.1016/s0278-6915(02)00220-x

19. Brinker TJ, Stamm-Balderjahn S, Seeger W, Groneberg DA. Education Against Tobacco (EAT): A quasiexperimental prospective evaluation of a programme for preventing smoking in secondary schools delivered by medical students: A study protocol. BMJ Open 2014;4(7):1-7. https://doi.org/10.1136/ bmjopen-2014-004909

20. Hamadeh RR, Ahmed J, Al-Kawari M, Bucheeri S. Quit tobacco clinics in Bahrain: Smoking cessation rates and patient satisfaction. Tob Induc Dis 2017;15(1):1-7. https://doi.org/10.1186/s12971-017-0115-1

21. Maziak W, Ward KD, Eissenberg T. Interventions for waterpipe smoking cessation. Cochrane Database Syst Rev 2007, Issue 4. Art. No. CD005549. https://doi.org/10.1002/14651858.cd005549

22. Maziak W, Jawad M, Jawad S, Ward KD, Eissenberg T, Asfar T. Interventions for waterpipe smoking cessation. Cochrane Database Syst Rev 2015, Issue 7. Art. No.: CD005549. https://doi. smoking cessation. Cochrane Databas
org/10.1002/14651858.CD005549.pub3

23. Jawad M, Jawad S, Waziry RK, Ballout RA, Akl EA. Interventions for waterpipe tobacco smoking prevention and cessation: A systematic review. Sci Rep 2016;11(6):25872. https://doi.org/10.1038/ srep 25872

24. Gardner K, Kearns R, Woodland L, et al. A scoping review of the evidence on health promotion interventions for reducing waterpipe smoking: Implications for practice. Front Public Health 2018;(6):117. https://doi.org/10.3389/fpubh.2018.00308

25. Moher D, Liberati A, Tetzlaff J, Altman DG. Preferred reporting items for systematic reviews and metaanalyses: The PRISMA statement. Ann Intern Med 2009;151(4):264-269. https://doi.org/10.7326/00034819-151-4-200908180-00135

26. Glasgow RE, Vogt TM, Boles SM. Evaluating the public health impact of health promotion interventions: The RE-AIM framework. Am J Public Health 1999;89(9):1322-1327. https://doi.org/10.2105/ ajph.89.9.1322

27. Stamm-Balderjahn S, Groneberg DA, Kusma B, Jagota A, Schönfeld N. Smoking prevention in school students: Positive effects of a hospital-based intervention. Dtsch Arztebl Int 2012;109(44):746-752. students: Positive effects of a hospital-basec
https://doi.org/10.3238/arztebl.2012.0746

28. Little MA, Talcott GW, Bursac Z, et al. Efficacy of a brief tobacco intervention for tobacco and nicotine containing product use in the US Air Force. Nicotine Tob Res 2015;18(5):1142-1149. https://doi.
. containing product use
org $/ 10.1093 / \mathrm{ntr} / \mathrm{ntv} 242$

29. Alavijeh FZ, Raisi Z, Asadollahi A, Irani RD, Kalhori SR. Impact of training high school female students in Ahvaz, Iran in the social skills required to avoid the use of drugs. Electron Physician 2016;8(5):23462354. https://doi.org/10.19082/2346

30. Lipkus IM, Eissenberg T, Schwartz-Bloom RD, Prokhorov AV, Levy J. Affecting perceptions of harm and addiction among college waterpipe tobacco smokers. Nicotine Tob Res 2011;13(7):599-610. https://doi. org $/ 10.1093 / \mathrm{ntr} / \mathrm{ntr} 049$
31. Dogar O, Jawad M, Shah SK, et al. Effect of cessation interventions on hookah smoking: Post-hoc analysis of a cluster-randomized controlled trial. Nicotine Tob Res 2013;16(6):682-688. https://doi.org/10.1093/ of a cluster-
$\mathrm{ntr} / \mathrm{ntt} 211$

32. Siddiqi K, Khan A, Ahmad M, et al. Action to stop smoking in suspected tuberculosis (ASSIST) in Pakistan: A cluster randomized, controlled trial. Ann Intern Med 2013;158(9):667-675. https://doi org/10.7326/0003-4819-158-9-201305070-00006

33. Thomas JL, Bengtson JE, Wang $\mathrm{Q}$, et al. Abstinence rates among college cigarette smokers enrolled in randomized clinical trial evaluating Quit and Win contests: The impact of concurrent hookah use. Pre Med 2015;76:20-25. https://doi.org/10.1016/j.ypmed.2015.03.010

34. Asfar T, Al Ali R, Rastam S, Maziak W, Ward KD. Behavioral cessation treatment of waterpipe smoking. The first pilot randomized controlled trial. Addict Behav 2014;39(6):1066-1074. https://doi.org/10.1016/j. addbeh.2014.02.012

35. Essa-Hadad J, Linn S, Rafaeli S. A web-based program to increase knowledge and reduce cigarette and nargila smoking among Arab university students in Israel: Mixed-methods study to test acceptability. J Med Internet Res 2015;17(2):1-12. https://doi.org/10.2196/jmir.2988

36. Mohlman MK, Boulos DN, El Setouhy, et al. A randomized, controlled community-wide intervention to reduce environmental tobacco smoke exposure. Nicotine Tob Res 2013;15(8):1372-1381. https://doi. org $/ 10.1093 /$ ntr $/$ nts 333

37. Mwaikambo L, Speizer IS, Schurmann A, Morgan G, Fikree F. What works in family planning Mwaikambo L, Speizer IS, Schurmann A, Morgan G, Fikree F. What works in family planning
interventions: A systematic review. Stud Fam Plann 2011;42(2):67-82. https://doi.org/10.1111/j.17284465.2011 .00267

38. Kassem NO, Kassem NO, Jackson SR, et al. Benzene uptake in hookah smokers and non-smokers attending hookah social events: Regulatory implications. Cancer Epidemiol Biomarkers Pre 2014;23(12):2793-2809. https://doi.org/10.1158/1055-9965.epi-14-0576

39. Zhou S, Behrooz L, Weitzman M, et al. Secondhand hookah smoke: An occupational hazard for hooka bar employees. Tob Control 2017;26(1):40-45. https://doi.org/10.1136/tobaccocontrol-2015-052505

40. Das JK, Salam RA, Arshad A, Finkelstein Y, Bhutta ZA. Interventions for adolescent substance abuse An overview of systematic reviews. J Adolesc Health 2016;59(4):61-75. https://doi.org/10.1016/j. jadohealth.2016.06.021

41. Bartholomew LK, Markham CM, Ruiter RAC, Fernàndez ME, Kok G, Parcel GS. Planning Health Promotion Programs: An Intervention Mapping Approach. 4th ed. Hoboken, NJ: John Wiley \& Sons 2016:50-150.

42. Ballbè M, Martínez C, Saltó E, et al. Maintenance of tobacco cessation programmes in public hospitals in Catalonia, Spain. Addict Behav 2015;42:136-139. https://doi.org/10.1016/j.addbeh.2014.11.028

43. South Africa. Tobacco Control Bill of South Africa. Government Gazette No. 41617, 9 May 2018:10-15. https://www.greengazette.co.za/documents/national-gazette-41617-of-09-may-2018vol-635_20180509-GGN-41617.pdf (accessed 29 April 2019).

Accepted 18 March 2019 